\title{
The use of Platelet-Rich Plasma in the Gynaecological Clinical Setting. A review.
}

\author{
Vasileios Sanoulis ${ }^{1}$, Nikolaos Nikolettos ${ }^{2}$, Nikolaos Vlahos ${ }^{3}$
}

${ }^{1}$ Aretaieion Hospital, Athens, Greece, ${ }^{2}$ Demokriteion University of Thrace, Alexandroupolis, Greece, ${ }^{3}$ Kapodistrian University of Athens, Athens, Greece

Correspondence

Correspondence should be addressed to Vasileios Sanoulis; vasanoulis@yahoo.gr

\section{Abstract}

Reports on clinical use of platelet-rich plasma (PRP) have dramatically increased in the last decade. PRP is a natural product where a high level of platelets is concentrated with growth factors concentration 3 to 5 times greater than plasma. In their alpha granules, platelets contain a cocktail of chemical mediators that trigger tissue regeneration, both through the promotion of angiogenesis and the initiation of cell regeneration. Such bioactive factors include platelet derived growth factor (PDGF), transforming growth factor-beta (TGF- $\beta$ ), vascular endothelial growth factor (VEGF), epidermal growth factor (EGF), fibroblast growth factor (FGF), insulin growth factor (IGF). In the gynaecological setting, PRP has been shown to facilitate wound healing and has been proposed as an effective treatment for the treatment of disorders such as Lichen Sclerosus (LS) or cervical ectopy. Evidence of PRP effectiveness in conditions of pelvic organ prolapse and urinary incontinence has also been reported. Recent publications have shown its positive effects in promoting endometrial and follicular growth and gestation in assisted reproduction cycles. PRP administration in the ovarian tissue has resulted in rejuvenation as evaluated by the relevant biochemical markers as well as reactivation of folliculogenesis. Other researchers have showed effective use in improving pregnancy outcomes in patients with repeated implantation failure. For managing repeated failures, several methods have been attempted, but recently PRP appears as effective in inducing endometrial development in patients with thin, poor endometrium. Another possible mechanism of PRP to endometrium is through its anti-inflammatory actions. PRP is an innovative therapeutic modality, as it is simple, easily performed, and effective, with minimal risk as it is an autologous product. The employment of regenerative medicine approaches will continue to grow in the gynaecological setting and the future trials should be carefully designed in order to facilitate the clinical translation.

Key words: Chronic Endomitritis, Frozen-Thawed Embryo Transfer, Growth Factors, Granulocyte-Colony Stimulating Factor, germline stem cells, Hepatocyte Growth Factor, Hormone Replacement Therapy, Intrauterine insemination, In Vitro Fertilization, lichen sclerosus, Premature Ovarian Failure, Platelet-Poor Plasma, Platelet-Rich Plasma, Repeated Implantation Failure, Very Small Embryonic like stem cells 


\section{Introduction}

Recent years have seen the blooming of regenerative medicine with the shift from the traditional approach of treatment to using the body's own cells and substances to enhance healing. In this context, platelet-rich plasma has been widely investigated and has played a protagonistic role in many clinical applications.

An exact definition for PRP is not absolutely clear; it is generally regarded as a blood derivate with a higher concentration of platelets compared to baseline whole blood levels ${ }^{1}$. Apart from PRP, other terms used in the literature to describe platelet-rich preparations from peripheral blood are platelet-rich growth factors, platelet-rich fibrin (PRF) matrix, PRF, and platelet concentrate (PC) ${ }^{2}$.

\section{History of PRP}

Kingsley first used the term PRP in 1954 to designate thrombocyte concentrate during experiments related to blood coagulation. In the clinical setting, it started to gain attention in the oral and maxillofacial field after 1998, when Marx and colleagues published their findings of using PRP with autologous bone grafts for the reconstruction of mandibular defects ${ }^{3}$. Clinical results in bone healing were promising, and, in time, PRP has been predominantly used in the musculoskeletal field. It has become one of the most studied orthobiologic agents in orthopaedics for bone, tendon, cartilage or muscle healing ${ }^{4}$. Nowadays, PRP represents a major breakthrough in the treatment of many medical conditions, including plastic surgery, maxillofacial surgery, dentistry and oral surgery, medical aesthetics, tissue engineering and research, cardiovascular surgery, orthopedic surgery and sports medicine, gastroenterology and urology,

\section{Biology - Science of PRP}

The biological rationale for the clinical use of PRP resides in the local delivery of growth factors (GFs), cytokines, chemokines, proteins and mediators - in physiological proportions - which contribute to the tissue healing and regeneration. This array of bioactive molecules is secreted by three types of granules (alpha, delta and lambda) located inside the platelets ${ }^{7-8}$.

GFs secreted by a-granules include platelet-derived growth factor (PDGF), epidermal growth factor (EGF), insulin-like growth factor (IGF), transforming growth factor $\beta$ (TGF- $\beta$ ), vascular endothelial growth factor (VEGF), hepatocyte growth factor (HGF), and fibroblast growth factor (FGF), which are involved in various mechanisms, including the enhancement of anabolism, bone and vessel remodeling, cell proliferation, angiogenesis, inflammation control, coagulation, and cell differentiation ${ }^{9}$. Alpha granules are also a source of cytokines, chemokines and proteins involved in stimulating chemotaxis, cell proliferation and maturation, modulating inflammatory molecules and attracting leukocytes ${ }^{1}$. Delta granules contain ADP, ATP, calcium, magnesium, adenosine and bioactive amines, such as serotine, histamine and dopamine. Lambda granules are the least studied; their main role is to remove cellular debris and infectious agents ${ }^{10}$.

Furthermore, the plasma itself contains molecules involved in the healing mechanism of connective tissues.

\section{Preparation and Composition}

There are many different commercial PRP systems available which simplify the preparation of PRP. Generally, whole blood is usually collected and mixed with an anticoagulant factor, prior to centrifugation, which separates red blood cells (RBCs) from plateletpoor plasma (PPP) and the "buffy coat," which contains the concentrated platelets and leukocytes. The platelet-concentrated layer is then isolated using several processing techniques and RBC and PPP layers may be discarded (11). In most cases, the prepa- 
ration devices achieve a PRP with platelet concentration of 2-5 folds the concentration in whole blood. Each commercial system has different isolation method (one- or two-step centrifugation), speed of centrifugation, and type of collection tube system and operation ${ }^{11}$. Excellent reviews have published the different PRP preparation methods, the classification of the end-products and the commercially

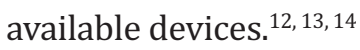

A recent review of the literature demonstrated that there is large heterogeneity among the different systems with regard to concentrations of platelets, leukocytes and growth factors ${ }^{14}$. There is an ongoing debate regarding the ideal volume of PRP to administer, the frequency of application, the exact site of administration, and which technique/preparation system to use ${ }^{2}$.

Before injecting PRP into the injured tissue, it can be activated ex vivo, with the use of calcium chloride and/or thrombin; otherwise, platelets are activated in situ by tissue factor, the initiator of the host response to injury. It has been reported that $70 \%$ of the GFs are secreted within ten minutes of activation. Within the first hour, the majority of GF release has occurred, although continued release occurs throughout the period of platelet viability (7 days $)^{7,8}$.

The first classification about platelet concentrates was proposed by Dohan Ehrenfest et al. and has since then been widely cited. It describes 4 main categories based on the cellular content (primarily leukocytes) and the fibrin architecture ${ }^{15}$ :

- Pure platelet-rich plasma (P-PRP), also known as leucocyte-poor PRP, lacks leucocytes and consists of a low-density fibrin network.

- Leucocyte and PRP (L-PRP), on the other hand, is prepared with leucocytes along with a fibrin network after activation.

- Pure platelet-rich fibrin (P-PRF), or leucocytepoor platelet-rich fibrin, do not contain leukocytes but present a high-density fibrin network.
- Lastly, leucocyte and platelet-rich fibrin (L-PRF), or second generation PRP, contains leukocytes and a high-density fibrin network.

P-PRP and L-PRP can be used as liquid solutions (injected) or in an activated gel form (placed on a skin wound or suture). P-PRF and L-PRF are in gel form and cannot be injected. They can be handled like a real solid material for other applications ${ }^{15}$.

\section{PRP in gynaecological applications PRP in wound healing}

The first prospective randomized controlled trial evaluating PRP in wound healing after caesarean section surgery has demonstrated positive effects; prior to skin closure, PRP was directly applied to the subcutaneous tissue of the wound site in 67 patients and results were compared to a control group of 71 patients who received the usual care. Eight weeks after surgery, patients treated with PRP showed a significantly greater reduction in Redness, Oedema, Ecchymosis, Discharge, Approximation (REEDA) scale (85.5\% vs. $72 \%$ of control, $p<0.001$ ). Significantly greater reduction was also demonstrated in pain, keloids, and hypertrophic scar formation for the PRP group ${ }^{16}$.

Significant reduction in pain on the day of surgery and on first postoperative day was published in a study by Fanning and colleagues ${ }^{17}$ on 55 patients undergoing gynaecologic surgeries such as hysterectomies and advanced urogynaecological and laparoscopic procedures, to whom autologous platelet grafts were directly applied to the surgical site.

Morelli and colleagues ${ }^{18}$ retrospectively analysed the results of 10 patients who had underwent radical vulvectomy and inguinofemoral node dissection and to whom platelet gel was applied on vaginal breach before vulvar reconstruction, and compared them to 15 patients who had the surgery without the platelet gel. Wound infection, wound breakdown, postoperative fever rate and duration of hospital stay were significantly decreased in the platelet gel group com- 
pared to surgery alone $(\mathrm{p}<0.032, \mathrm{p}<0.048, \mathrm{p}<0.001$, $\mathrm{p}<0.001$ respectively). In the same study, average time for wound healing was found to be 23.6 days whereas for the control group it was 93.3 days $(\mathrm{p}<0.001)$.

\section{Lichen Sclerosus}

Lichen Sclerosus (LS) is a chronic inflammatory dermatological condition which mainly affects the anogenital region but can also be generalised. The aetiology and pathogenesis of LS is not elucidated; evidence points to a genetic predisposition and autoimmunity as a pathogenic mechanism. All of current treatments of LS are aimed at controlling the symptoms and there is not yet a cure for the disease. Corticosteroid administration is the therapy of choice but it is a regime of high-maintenance with variable results and low rates of compliance. PRP has been studied as a treatment option for women with $\mathrm{LS}^{19,20}$.

Behnia-Willison et $\mathrm{al}^{21}$ studied the application of PRP in 28 LS patients, unresponsive to topical steroid treatment. Using a 27-gauge needle, they injected PRP in a fanning pattern to any affected areas of the external genitalia, including the labia majora, labia minora, clitoris, and clitoral hood. Patients received the treatment three times, 4 to 6 weeks apart and again at 12 months. Nearly all patients exhibited clinical improvements in the size of their lesions with minimal pain and no complications. In $28.6 \%$ of the cases, the lesions completely disappeared. Due to the small number of patients in the study and the fact that most were postmenopausal, it is difficult to generalize findings to women of reproductive age. The authors concluded that PRP injections could be considered an effective alternative to corticosteroids for the treatment of vulvovaginal autoimmune conditions such as LS.

\section{Cervical ectropion}

Cervical ectropion (or else cervical ectopy or erosion) occurs when eversion of the endocervix ex- poses epithelium to the vaginal milieu. Reported treatment modalities include laser ablation, cryotherapy, ultrasound therapy, microwave ablation and, more recently, $\mathrm{PRP}^{22,23}$. Hua et al. ${ }^{24}$ reported the effectiveness of autologous PRP for the treatment of benign cervical ectopy. The study group consisted of 120 women who were randomised to either laser or PRP therapy. No statistical difference was found for the complete cure rate between the two groups (93.7\% and $92.4 \%$ for PRP and laser respectively). However, the mean time to re-epithelialisation was significantly shorter in the PRP group $(\mathrm{p}<0.01)$.

\section{Urogenital applications}

Shirvan et al. ${ }^{25}$ employed PRP to treat vesicovaginal fistula in 12 patients and concluded that PRP injection and PRF glue interposition in the tract constitute a safe and effective approach for the fistula closure. PRP combined with mucosal advancement flap was used in a small pilot trial to treat Crohn's disease-related high perianal fistulas ${ }^{26}$. This involved placement of non-cutting seton for drainage of the fistula tract followed by local treatment with PRP and mucosal advancement flap. At 1-year follow-up, seven of the ten patients had a dry fistula, a favourable healing rate compared to the $40-50 \%$ for the mucosal advancement flap reported in other studies.

In vaginal surgery, PRF has been proposed to act as a graft material with better healing and functional outcomes. In a study of 10 patients requiring surgery for prolapse recurrence, the success rate was $80 \%$, prolapse symptoms improved by $100 \%$ and sexual activity increased by $20 \%$ without dyspareunia ${ }^{27}$.

Two recent articles summarized evidence regarding the use of PRP in pelvic organ prolapse and urinary incontinence. They found that PRP helps to regulate tissue reconstruction and the restoration of ligament strength; however, more animal experimental and clinical studies are needed to justify its use and elucidate the role of $\mathrm{PRP}^{28,29}$. 


\section{PRP in Reproductive Medicine}

With the aim to investigate PRP effectiveness in the management of poor responder patients, Sfakianoudis, Simopoulou, Nitsos, Rapani, Pantou, et al. ${ }^{30}$ employed non-surgical autologous PRP ovarian infusions to treat 3 extreme poor responder patients, with recurrent cancelled cycles, poor oocyte yield following stimulation, high follicle-stimulating hormone (FSH) and low Anti-Müllerian hormone (AMH) levels. Five $\mathrm{mL}$ of PRP was infused into each ovary using a non-surgical, transvaginal ultrasound-guided multifocal intramedullary injection and diffusion in the subcortical layers. Prior to the PRP treatment, FSH and AMH were measured on the third day of the menstrual cycle immediately before the treatment while after the PRP treatment the measurements were done on the third day of the directly subsequent menstrual cycle. The authors recorded a mean decline of $67.33 \%$ in FSH and an increase of $75.18 \%$ in AMH following PRP administration, a change which strongly indicated an improvement in ovarian functionality. Natural IVF cycles were performed subsequent to PRP ovarian infusion to all 3 patients. A natural conception was reported at 24 weeks, an uncomplicated healthy pregnancy at 17 weeks and a successful live birth. Enhanced oocyte, and therefore, embryo quality has been suggested by the authors as the underlying factors permitting the successful pregnancies. They concluded that prospective wellcontrolled clinical trials on the effect of ovarian PRP treatment particularly on AMH levels are required to ascertain validity ${ }^{30}$.

The same research team employed PRP for the treatment of a 40-year-old patient diagnosed with premature menopause at the age of $35^{31}$. The FSH levels were recorded at $149 \mathrm{mIU} / \mathrm{mL}$ and the antiMüllerian hormone (AMH) levels were $0.02 \mathrm{ng} / \mathrm{mL}$. Approximately $4 \mathrm{~mL}$ of PRP, with concentration of 900,000 platelets $/ \mu$ l, were administered per ovary through intraovarian injection. Menstruation reoc- curred 6 weeks post injection and FSH and AMH were recorded at $27 \mathrm{mIU} / \mathrm{mL}$ and $0.08 \mathrm{ng} / \mathrm{mL}$ respectively. On day 16 of the menstrual cycle, and after the administration of human chorionic gonadotrophin (hCG) on day 14, oocyte collection was performed with subsequent insemination through intracytoplasmic sperm injection (ICSI). Following the embryo transfer, a biochemical pregnancy was confirmed by a positive hCG level of $42 \mathrm{mIU} / \mathrm{mL}$. This was the first report of a PRP application leading to pregnancy in menopause ${ }^{31}$.

Sfakianoudis and colleagues further decided to employ PRP so as to tackle persistent CE after ineffective antibiotic schemes ${ }^{32}$. The 35-year-old woman of their study had premature ovarian insufficiency and a history of 6 failed donated embryo transfers. Following appropriate antimycotic and hormonal treatment, she underwent a new embryo transfer, but with subsequent pregnancy loss. Following a second antibiotic scheme, persistent CE was diagnosed and increased inflammation was shown through the SEM images. PRP therapy was then employed; $2.5 \mathrm{ml}$ of PRP was infused into the uterine cavity. After confirming no signs of CE through microbiological analysis and SEM, the patient underwent another embryo transfer of two donated blastocysts which led to clinical pregnancies and live births. In the aforementioned studies, the clinical team reported a final concentration of $900,000 / \mu \mathrm{l}$ in the PRP sample ${ }^{32}$.

\section{Thin endometrium}

Endometrial receptivity has been acknowledged as a key factor for the success of IVF. The role of endometrial thickness has been extensively discussed; a thin endometrium is generally regarded to be associated with lower implantation rates, although there have been researchers who could not establish a relationship between endometrial thickness and IVF clinical outcomes. When a positive correlation was reported, a value below 6-8 $\mathrm{mm}$ was considered 
a negative predictive indicator for IVF outcomes; there is no consensus though as to the exact threshold for the endometrium to be defined as thin. Instead of studying IVF outcomes above and below a cut-off thickness value, Liu et al..$^{33}$ investigated the impact of gradient decline in thickness. They analysed 21,914 fresh and 18,942 frozen-thaw IVFET cycles and found that clinical pregnancy and live birth rates decreased significantly for each $\mathrm{mm}$ increment below $8 \mathrm{~mm}$ in the fresh IVF-ET group and below $7 \mathrm{~mm}$ in the frozen-ET group.

In addition to the lower probability of clinical pregnancy, a thin endometrium appears to be associated with both early and late pregnancy complications. In a study of Yuan et al. ${ }^{34}$ of 10,787 cycles, the miscarriage rate was as high as $26.7 \%$ in patients who conceived with IVF and had an endometrial thickness $\leq 8 \mathrm{~mm}$. The rate decreased with increasing endometrial thickness $(26.7 \%$ vs. $23.8 \%$ vs. $19.9 \%$ vs. $17.5 \%$ for EMT $<8.0 \mathrm{~mm}$ vs. $8.0-11.0 \mathrm{~mm}$ vs. $11.1-15.0 \mathrm{~mm}$ vs. $>15 \mathrm{~mm}$, respectively) but it reached statistical significance only between 8.0$11.0 \mathrm{~mm}$ vs. $11.1-15.0 \mathrm{~mm}$ (34).

Liu et al. (33) reported that the miscarriage rate in fresh IVF cycles significantly increased with every $\mathrm{mm}$ decrease in thickness below $8 \mathrm{~mm}$ (22\% vs. $26.4 \%$ vs. $27.1 \%$ vs. $30 \%$; $\mathrm{p}=0.01$ for $\mathrm{EMT} \geq 8 \mathrm{~mm}$ vs. 7.0-7.9 mm vs. $6.0-6.9 \mathrm{~mm}$ vs. $5.0-5.9 \mathrm{~mm}$ respectively). The difference did not reach statistical significance in the frozen-ET cycles ( $p=0.09)$.

Much research has focused on the challenging objective to improve endometrial growth in an effort to increase implantation and live birth rates in ART; treatment options include hormonal adjustment through the long-term administration of estrogen, human chorionic gonadotropin (hCG) and gonadotropin-releasing hormone $(\mathrm{GnRH})^{35}$ - , transvaginal endometrial perfusion of granulocyte colony stimulating factor (G-CSF), vaginal sildenafil application, low-dose of aspirin, pentoxifylline (PTX) and to- copherol (vitamin E) and electroacupuncture ${ }^{36,37}$. There is lack of solid evidence in favour of one option over the other, and, still, a considerable number of women with thin endometrium have no response. The need for novel, alternative treatments has led to the investigation of stem cell therapy, which appears to be promising in the first human clinical studies but its safety and effectiveness has to be further evaluated, especially because of its high cost and complex clinical application ${ }^{37,38,39}$.

PRP application on the other hand is a simple, relatively low-cost and minimally invasive procedure which has been recently explored for the management of refractory endometrium. The first report of the clinical use of PRP in thin endometrium was in 2015 by Chang and colleagues ${ }^{40}$. Five patients with a history of the thin endometrium (on the day of hCG administration) were recruited in the study. PRP was infused into the uterine cavity on the 10th day of FET cycle. If endometrial thickness failed to increase 72 $\mathrm{h}$ later, PRP infusion was done 1-2 times in each cycle. When the endometrium thickness reached $>7$ mm embryos were transferred. Successful endometrial expansion and pregnancy were observed in all the patients after PRP infusion. The same research group further investigated the effect of PRP in a larger number of patients (41). They conducted a prospective cohort study including 64 patients with endometrium thickness $<7 \mathrm{~mm}$ and previously cancelled frozen ET cycles. PRP was administered via intrauterine catheter on the 10th day in HRT cycle and on progesterone administration day. After PRP treatment, mean endometrium thickness in PRP group (34 patients) was $7.65 \mathrm{~mm}$, which was significantly higher $(\mathrm{p}<0.05)$ than that in control group (30 patients) $(6.52 \mathrm{~mm})$. Clinical pregnancy and implantation rates were also significantly $(\mathrm{p}<0.05)$ higher in the PRP group ( $44.12 \%$ and $27.94 \%$ vs. $20 \%$ and $11.67 \%$ respectively). On what concerns the PRP sample characteristics, the average platelet concen- 
tration increase was 4.21-fold, with concentrations of PDGF-AB, PDGF-BB, and TGF-b being much higher in PRP compared with whole blood $(33 \mathrm{ng} / \mathrm{ml}$ vs. $10 \mathrm{ng} / \mathrm{mL}, 6.6 \mathrm{ng} / \mathrm{ml}$ vs. $1.5 \mathrm{ng} / \mathrm{mL}, 30 \mathrm{ng} / \mathrm{mL}$ vs. 13ng/mL, respectively; $\mathrm{P}<.01)^{41}$.

In a randomized clinical trial by Eftekhar et al. ${ }^{42}$, including 83 women, endometrial thickness increased to $8.67 \pm 0.64 \mathrm{~mm}$ in the 40 patients who underwent PRP treatment, a statistically significant difference from the control (43 patients). The implantation rate and per-cycle clinical pregnancy rate were significantly higher in PRP group. Cycle cancellation rate was lower in PRP group but not significant (7 in PRP group and 10 in controls, $p=0.061$ ).

\section{Sexual dysfunction}

The injection of PRP into the anterior vaginal wall and the clitoris orgasmic to treat sexual dysfunction is called ' $\mathrm{O}$-shot', a term patented by Runels who first published the results of this technique in a pilot study enrolling 11 patients presenting with complaints associated with female orgasmic disorder, hypoactive sexual arousal disorder, anorgasmia, or dyspareunia ${ }^{43}$. The Female Sexual Function (FSF) Index and the Female Sexual Distress Scale, were used to measure the response to this therapeutic intervention. The mean Distress score dropped from 17 to 7 ( $p=0.04$ ) while the improvement in the FSF scale was 5.5 points $(\mathrm{p}=0.01)$. The authors suggested that PRP intravaginal and intraclitoral injections could be an effective method to treat certain types of female sexual dysfunction, especially in the areas of desire, arousal, lubrication and orgasm ${ }^{43}$.

Vaginal rejuvenation procedures for the treatment of vaginal laxity and the improvement of sexual functioning are gaining increasing popularity, especially non-traditional, minimally invasive approaches such as fractional $\mathrm{CO}_{2}$ laser or radiofrequency treatments ${ }^{44}$. One novel technique described by Aguila and colleagues ${ }^{45}$ involved vulvovaginal lipofilling (16
$\mathrm{mL}$ of purified fat cells) of the posterior vaginal wall and injection of a mixture of hyaluronic acid and PRP (10 mL of combined solution) subcutaneously in the perineum area. At 3 months follow-up, the patient was satisfied with the result, reporting a score of $30 / 40$ at the Stabbatsberg self-rating scale, compared to $15 / 40$ prior to intervention. Clinical examination showed improved vaginal trophicity and a diminished vaginal calibre. In another study, autologous fat ( $36 \mathrm{~mL}$ ) mixed with autologous PRP ( $4 \mathrm{~mL}$ ) was injected into the subcutaneous layer of the labia majora of a 67-year-old woman with vaginal atrophy. One month after injection, clinical symptoms of vaginal pruritus and irritation, which had been present for over 5 years before treatment, had been disappeared, while volume augmentation of the labia majora was evident and missing fullness and tone of labia majora were corrected. The restoration of contour was maintained well after 1 year ${ }^{45}$.

\section{Mechanism of action}

PRP administration, through the release of the biologically active substances, is proposed to assist in tissue regeneration, the enhancement of anabolic signalling pathways, cell differentiation and proliferation, angiogenesis initiation and control.

Animal model studies in the reproductive context have reported that PRP enhances primordial and primary preantral follicles development as well as prevents possible ischemia following ovarian injury. Through administration of PRP, cytokines and growth factors necessary for enhancing endometrial receptivity and improving implantation rate, are secreted. At the endometrial site these biomolecules activate stromal cells and recruit leukocytes to promote angiogenesis and induce repair mediated by cell proliferation and migration. Recent in vitro evidence provided novel information that PRP enhances migration and proliferation of human endometrial epithelial (Ishikawa) cells, endometrial 
stromal fibroblasts, endometrial mesenchymal stem cells ${ }^{46,47}$.

PRP's anti-microbial and anti-inflammatory properties have been demonstrated on human chondrocytes and on equine and bovine uterine infections, and may explain the favourable environment for the endometrial growth and receptivity ${ }^{48}$. Moreover, since the implantation process relies on an inflammatory/anti-inflammatory equilibrium, a possible mechanism of PRP action on receptivity of endometrium is via anti-inflammatory action of factors like $\mathrm{HGF}^{49}$.

Another piece of evidence in the role of PRP in the reproductive field may be given by the member of the TGF-b superfamily, growth differentiation factor 9 (GDF-9), which is contained in PRP and is regarded as a biomarker of oocyte maturation potential ${ }^{31}$.

In the study of Chang et al. ${ }^{41}$, the platelet concentration in PRP was 4.21-folds higher than baseline and significantly higher concentrations of PDGF-AB, PDGF-BB, and TGF-b were also recorded. (33 ng/ml vs. $10 \mathrm{ng} / \mathrm{mL}, 6.6 \mathrm{ng} / \mathrm{ml}$ versus $1.5 \mathrm{ng} / \mathrm{mL}, 30 \mathrm{ng} / \mathrm{mL}$ versus $13 \mathrm{ng} / \mathrm{mL}$, respectively, all $\mathrm{p}<.01$ ). They suggested that PRP with high concentration of growth factors and cytokines can stimulate the mitogenesis and proliferation of endometrial cells or endometrial stem cells, and then activate endocrine-paracrine pathways for improving the endometrial response to promote embryo implantation and pregnancy. Aguilar et al. ${ }^{45}$, through a different preparation protocol, reported a platelet dose of 0.9 billion and concentrations of TGF-b and PDGF-AB to be 67.37 $\mathrm{ng} / \mathrm{mL}$ and $12.71 \mathrm{ng} / \mathrm{mL}$, respectively.

Pain reduction observed with the use of PRP in the wound management after caesarean section surgery is thought to be explained by the action of serotonin released from platelets ${ }^{16}$.

Studies have also shown variation of platelet, leukocyte, growth factor and cytokine levels across PRP preparation methods. Since a specific platelet dose may be required to achieve a clinical effect, it is important to identify the preparation protocols that produce different doses ${ }^{50}$. PRP characteristics are unfortunately not included in the methods of the most published research articles, especially in the gynaecological field, in which only recently did the scientific community start to explore its full potential.

\section{Conclusion}

PRP is a simple, efficient and minimally invasive method for obtaining a natural concentration of autologous growth factors and biologically active molecules with strong potential for improving tissue healing and promoting regeneration. In addition, due to its autologous origin and minimally invasive collection technique, the risks of infection or immune reactions are minimised. Heterogenous processing methods, the lack of standardisation in the nomenclature and the various classifications render the interpretation of the literature regarding the clinical effectiveness challenging. The employment of regenerative medicine approaches will continue to grow in the gynaecological setting and the future trials should be carefully designed in order to facilitate the clinical translation.

\section{References}

1. Kon E, Filardo G, Di Matteo B, Marcacci M. PRP for the treatment of cartilage pathology. Open Orthop J 2013; 7:120-8.

2. Alves R, Grimalt R: A Review of Platelet-Rich Plasma: History, Biology, Mechanism of Action, and Classification. Skin Appendage Disord 2018; 4:18-24.

3. Ehrenfest DM, Bielecki T, Mishra A, et al. In search of a consensus terminology in the field of platelet concentrates for surgical use: platelet-rich plasma (PRP), platelet-rich fibrin (PRF), fibrin gel polymerization and leukocytes. Curr Pharm Biotechnol 2012; 13:1131-7. 
4. Hussain N, Johal H, Bhandari M. An evidencebased evaluation on the use of platelet rich plasma in orthopedics - a review of the literature. SICOT J 2017; 3:57.

5. Andia E, Rubio-Azpeitia J, Martin I, Abate M: Current concepts and translational uses of platelet rich plasma biotechnology; in Ekinci D (ed.): Biotechnology. InTech, 2015, DOI: 10.5772/ 59954. Retrieved from: https://www. intechopen.com/books/biotechnology/current-concepts-and-translational-uses-of-platelet-rich-pla sma-biotechnology.

6. Hara RG \& Basu T. Platelet-rich plasma in regenerative medicine. Biomedical Research and Therapy 2014; 1(01):25-31. Retrieved from: http:// www.bmrat.org/index.php/BMRAT/article/vie $\mathrm{w} / 14$

7. Cole BJ, Seroyer ST, Filardo G, Bajaj S, Fortier LA. Platelet-rich plasma: where are we now and where are we going? Sports Health 2010; 2(3): 203-10.

8. Pavlovic V, Ciric M, Jovanovic V, Stojanovic P. Platelet Rich Plasma: a short overview of certain bioactive components. Open Med (Wars) 2016; 11(1):242-7.

9. Salamanna F, Veronesi F, Maglio M, Della Bella E, Sartori M, Fini M. New and emerging strategies in platelet-rich plasma application in musculoskeletal regenerative procedures: general overview on still open questions and outlook. Biomed Res Int 2015; 2015:846045.

10. Boswell SG, Cole BJ, Sundman EA, Karas V, Fortier LA. Platelet-rich plasma: a milieu of bioactive factors. Arthroscopy 2012;28: 429-39.

11. Le ADK, Enweze L, DeBaun MR, Dragoo JL. Platelet-Rich Plasma. Clin Sports Med 2019; 38:17-44.

12. DeLong JM, Russell RP, Mazzocca AD. Plateletrich plasma: The PAW classification system. Arthroscopy 2012; 28:998-1009.
13. Mautner K, Malanga GA, Smith J, Shiple B, Ibrahim V, Sampson S, Bowen JE. A call for a standard classification system for future biologic research: The rationale for new PRP nomenclature. PM R 2015; 7:S53-S59.

14. Oudelaar BW, Peerbooms JC, Huis In 't Veld R, Vochteloo AJH. Concentrations of Blood Components in Commercial Platelet-Rich Plasma Separation Systems: A Review of the Literature. Am J Sports Med 2019; 47(2):479-87.

15. Ehrenfest DM, Rasmusson L, Albrektsson T. Classification of platelet concentrates: from pure platelet-rich plasma (P-PRP) to leucocyte- and platelet-rich fibrin (L-PRF) Trends Biotechnol 2009; 27:158-67.

16. Tehranian A, Esfehani-Mehr B, Pirjani R, Rezaei $N$, Sadat Heidary S, Sepidarkish M. Application of Autologous Platelet-Rich Plasma (PRP) on Wound Healing After Caesarean Section in HighRisk Patients. Iran Red Crescent Med J 2016; 18(7):e34449.

17. Fanning J, Murrain L, Flora R, Hutchings T, Johnson JM, Fenton BW. Phase I/II prospective trail of autologous platelet tissue graft in gynecologic surgery. J Minim Invasive Gynecol 2007; 14:633-7.

18. Morelli M, Rocca ML, Venturella R, Di Cello A, Del Negro S, Condorelli M, et al. Adjuvant use of platelet gel for wound breakdown prevention in advanced vulvar cancer surgery: a retrospective study. Int J Gynecol Cancer 2013; 23:1490-4.

19. Halonen P, Jakobsson M, Heikinheimo O, Riska A, Gissler M, Pukkala E. Lichen sclerosus and risk of cancer. Int J Cancer 2017; 140:1998-2002.

20. Kirtschig G. Lichen Sclerosus-Presentation, Diagnosis and Management. Dtsch Arztebl Int 2016; 113(19):337-43. doi:10.3238/arztebl.2016.0337

21. Behnia-Willison F, Pour NR, Mohamadi B, et al. Use of Platelet-rich Plasma for Vulvovaginal Autoimmune Conditions Like Lichen Sclerosus. Plast Reconstr Surg Glob Open 2016; 
4(11):e1124.

22. Casey PM, Long ME, Marnach ML. Abnormal cervical appearance: what to do, when to worry? Mayo Clin Proc. 2011; 86(2):147-51.

23. Mitchell L, King M, Brillhart H, Goldstein A. Cervical Ectropion May Be a Cause of Desquamative Inflammatory Vaginitis. Sex Med 2017; 5(3):e212-e214.

24. Hua X, Zeng Y, Zhang R, Wang H, Diao J, Zhang P. Using platelet rich plasma for the treatment of symptomatic cervical ectopy. Int J Gynaecol Obstet 2012; 119:26-9.

25. Shirvan MK, Alamdari DH, Ghoreifi A. A novel method for iatrogenic vesicovaginal fistula treatment: autologous platelet rich plasma injection and platelet rich fibrin glue interposition. J Urol 2013; 189:2125-9.

26. Göttgens KW, Smeets RR, Stassen LP, Beets GL, Pierik M, Breukink SO. Treatment of Crohn's disease-related high perianal fistulas combining the mucosa advancement flap with platelet-rich plasma: a pilot study. Tech Coloproctol 2015; 19(8):455-9.

27. Gorlero F, Glorio M, Lorenzi P, Bruno-Franco M, and Mazzei C. New approach in vaginal prolapse repair: miniinvasive surgery associated with application of platelet-rich fibrin. International Urogynecology Journal 2012; 23(6): 715-22.

28. Chrysanthopoulou EL, Pergialiotis V, Perrea D, Kourkoulis S, Verikokos C, Doumouchtsis SK. Platelet rich plasma as a minimally invasive approach to uterine prolapse. Med Hypotheses 2017; 104:97-100.

29. Nikolopoulos KI, Pergialiotis V, Perrea D, Doumouchtsis SK. Restoration of the pubourethral ligament with platelet rich plasma for the treatment of stress urinary incontinence. Med Hypotheses 2016; 90:29-31.

30. Sfakianoudis K, Simopoulou M, Nitsos N, Rapani A, Pantou A, Vaxevanoglou T, Kokkali G, Kout- silieris M, Pantos K: A Case Series on PlateletRich Plasma Revolutionary Management of Poor Responder Patients. Gynecol Obstet Invest 2019; 84:99-106.

31. Sfakianoudis K, Simopoulou M, Nitsos N, Rapani A, Pappas A, Pantou A, Chronopoulou, M, Deligeoroglou E, Koutsilieris M, Pantos K. Autologous Platelet-Rich Plasma Treatment Enables Pregnancy for a Woman in Premature Menopause. J Clin Med 2019; 8:1.

32. Sfakianoudis K, Simopoulou M, Nitsos N, Lazaros L, Rapani A, Pantou A, Koutsilieris M, Nikas Y, Pantos K. Successful Implantation and Live Birth Following Autologous Platelet-rich Plasma Treatment for a Patient with Recurrent Implantation Failure and Chronic Endometritis. In Vivo 2019; 33(2):515-21.

33. Liu KE, Hartman M, Hartman A, Luo ZC, Mahutte $\mathrm{N}$. The impact of a thin endometrial lining on fresh and frozen-thaw IVF outcomes: an analysis of over 40000 embryo transfers. Hum Reprod 2018; 33(10):1883-8.

34. Yuan X, et al. Endometrial thickness as a predictor of pregnancy outcomes in 10787 fresh IVFICSI cycles. J Reproductive Biomedicine Online 2016; 33:197-205.

35. Wu Y, Gao X, Lu X, et al. Endometrial thickness affects the outcome of in vitro fertilization and embryo transfer in normal responders after $\mathrm{GnRH}$ antagonist administration. Reprod Biol Endocrinol 2014; 12:96.

36. Garcia-Velasco JA, Acevedo B, Alvarez C, Alvarez M, Bellver J, Fontes J, Landeras J, Manau D, Martinez F, Muñoz E, Robles A, Rodriguez-Tabernero L. Strategies to manage refractory endometrium: state of the art in 2016. Reprod Biomed Online 2016; 32(5):474-89.

37. Mouhayar Y, Sharara FI. G-CSF and stem cell therapy for the treatment of refractory thin lining in assisted reproductive technology. J Assist Reprod 
Genet 2017; 34(7):831-7.

38. Santamaria X, Cabanillas S, Cervello I, Arbona C, Raga F, Ferro J, et al. Autologous cell therapy with CD133+ bone marrow-derived stem cells from refractory Asherman's syndrome and endometrial atrophy: a pilot cohort study. Hum Reprod 2016; 31(5):1087-96.

39. Singh N, Mohanty S, Seth T, ShankarM, Bhaskaran S, Dharmendra S. Autologous stem cell transplantation in refractory Asherman's syndrome: a novel cell based therapy. J Hum Reprod Sci 2014; 7(2):93-8.

40. Chang Y, J. Li, Y. Chen, L. Wei, X. Yang, Y. Shi, et al. Autologous platelet-rich plasma promotes endometrial growth and improves pregnancy outcome during in vitro fertilization Int J Clin Exp Med 2015; 8(1):1286.

41. Chang Y, Li J, Wei LN, Pang J, Chen J, Liang X. Autologous platelet-rich plasma infusion improves clinical pregnancy rate in frozen embryo transfer cycles for women with thin endometrium. Medicine (Baltimore) 2019; 98(3):e14062.

42. Eftekhar M, Neghab N, Naghshineh E, Khani P. Can autologous platelet rich plasma expand endometrial thickness and improve pregnancy rate during frozen-thawed embryo transfer cycle? A randomized clinical trial. Taiwan J Obstet Gynecol 2018; 57(6):810-3.

43. Runels C, Melnick H, Debourbon E, Roy L. A pilot study of the effect of localized injections of autologous platelet rich plasma (PRP) for the treatment of female sexual dysfunction. J Womens Health Care 2014; 3:169.

44. Barbara G, Facchin F, Buggio L, Alberico D, Frattaruolo MP, Kustermann A. Vaginal rejuvenation: current perspectives. Int J Womens Health 2017; 9:513-9.
45. Aguilar P, Hersant B, SidAhmed-Mexi M, Bosc R, Vidal L, Menigaud JP. Novel technique of vulvovaginal rejuvenation by lipofilling and injection of combined platelet-rich-plasma and hyaluronic acid: a case-report. Springerplus 2016; 5(1):1184.

46. Aghajanova L, Houshdaran S, Balayan S, Manvelyan E, Irwin J., Huddleston HG, Giudice LC. In vitro evidence that platelet-rich plasma stimulates cellular processes involved in endometrial regeneration. J Assist Reprod Genet 2018; 35:757-70.

47. Makieva S, Giacomini E, Ottolina J, Sanchez AM, Papaleo E, Viganò P. Inside the Endometrial Cell Signaling Subway: Mind the Gap(s). Int J Mol Sci 2018; 19(9):2477.

48. Bos-Mikich A, de Oliveira R, Frantz N. Plateletrich plasma therapy and reproductive medicine. J Assist Reprod Genet 2018; 35(5):753-6.

49. Mehrafza M, Kabodmehri R, Nikpouri Z, et al. Comparing the Impact of Autologous Plateletrich Plasma and Granulocyte Colony Stimulating Factor on Pregnancy Outcome in Patients with Repeated Implantation Failure. J Reprod Infertil 2019; 20(1):35-41.

50. Fitzpatrick J, Bulsara MK, McCrory PR, Richardson MD, Zheng MH. Analysis of Platelet-Rich Plasma Extraction: Variations in Platelet and Blood Components Between 4 Common Commercial Kits. Orthop J Sports Med 2017; 5(1):2325967116675272.
Received 3-5-2019

Revised 20-5-2019

Accepted 4-6-2019 